\title{
Technology Based Treatment for UreteroPelvic Junction Obstruction
}

\author{
Weil R. Lai, MD, Carrie A. Stewart, MD, and Raju Thomas, MD, FACS, MHA
}

\begin{abstract}
Surgical management of ureteropelvic junction obstruction (UPJO) has historically been performed with open pyeloplasty. With the advent of endourology, laparoscopy, and robotics, minimally-invasive techniques have been described and accepted as alternatives to open surgery. Each of these approaches has its own advantages and disadvantages, equipment needs, degree of invasiveness, and experience of the treating urologist. Advocates and critics have their own say as to their preferred technique. In this article, we review the chronological evolution of these techniques and discuss their current role in the management of UPJO.
\end{abstract}

Keywords: obstruction, percutaneous renal surgery, ureteroscopy, laparoscopy approach, robotics

\section{Introduction}

$\mathbf{M}$ ANAGEMENT OF URETEROPELVIC JUNCTION obstruction (UPJO) has recently evolved through a virtual menu of treatment choices. Before this, the only option available was open surgical pyeloplasty. Though effective in the treatment of UPJO, it has been an invasive procedure and, therefore, associated with considerable morbidity and associated complications, especially in the adult population. With the introduction of endourologic techniques in the 1980s, less invasive techniques have been developed to minimize morbidity while attempting to achieve similar outcomes as open pyeloplasty (OP). With the popularization of laparoscopy and robotics in the 1990s and 2000s, respectively, there has been a shift back to performing pyeloplasty, although less invasively. Thus, this led to the emergence of the menu options mentioned earlier. We will review the chronological evolution of these techniques and discuss their current role in treating UPJO (Fig. 1) under different and varied presentations.

\section{Antegrade Endopyelotomy}

The earliest endourologic techniques in endopyelotomy were built on the principles and advances in the field of endourology, especially in percutaneous access to the kidney and percutaneous nephrostolithotripsy (PCNL). In 1983, Wickham and Kellet presented their initial experience of antegrade endopyelotomy in three patients with their "percutaneous pyelolysis" technique. ${ }^{1}$ This was soon followed by their 1984 case series of 28 patients, with a success rate of 64\%. ${ }^{2}$ In 1986, Arthur Smith, an early pioneer in endourology, presented his series of 31 patients who underwent antegrade endopyelotomy, with a success rate of $87 \% .^{3}$ Of those 31 patients, 12 had concomitant PCNL, and 8 had prior unsuccessful OP.

The technique of antegrade endopyelotomy entails percutaneous access to the kidney, preferably through an upper pole access, giving the urologist direct access to the ureteropelvic junction (UPJ) (Fig. 2). Results have shown that this procedure is best suited for a short-segment UPJO, and the antegrade incision for endopyelotomy is made in a lateral position, thereby avoiding injury to any crossing vessels. ${ }^{4}$ Techniques for incisions include cold-knife, electrocautery, and laser-assisted procedures. ${ }^{5,6}$ Depending on the surgeon and experience, the incised UPJ is subject to balloon dilatation and stenting. The type, size, and duration of stenting are operator dependent.

The success rate of antegrade endopyelotomy varies depending on the series, follow-up duration, and definition of success. In Gerber and Lyon's meta-analysis on the outcomes of 672 patients across 12 centers who underwent antegrade endopyelotomy, they reported success rates ranging from $57 \%$ to $100 \%{ }^{7}$ with follow-up intervals ranging from 2 to 96 months. Success rates are known to decrease with time in these patients. In 1997, Arthur Smith published his largest series of 401 antegrade endopyelotomies that had been accumulated over 12 years of practice. ${ }^{8}$ With a mean follow-up of 51 months, overall success rate was $85 \%$. Poor renal function (defined as less than $25 \%$ of total renal function) and highgrade hydronephrosis were identified as independent factors associated with treatment failure, with corresponding drops in success rates to $54 \%$ and $50 \%$, respectively, over the long term.

In a retrospective study comparing long-term success of antegrade endopyelotomy with open/laparoscopic pyeloplasty

Department of Urology, Tulane University School of Medicine, New Orleans, Louisiana. 


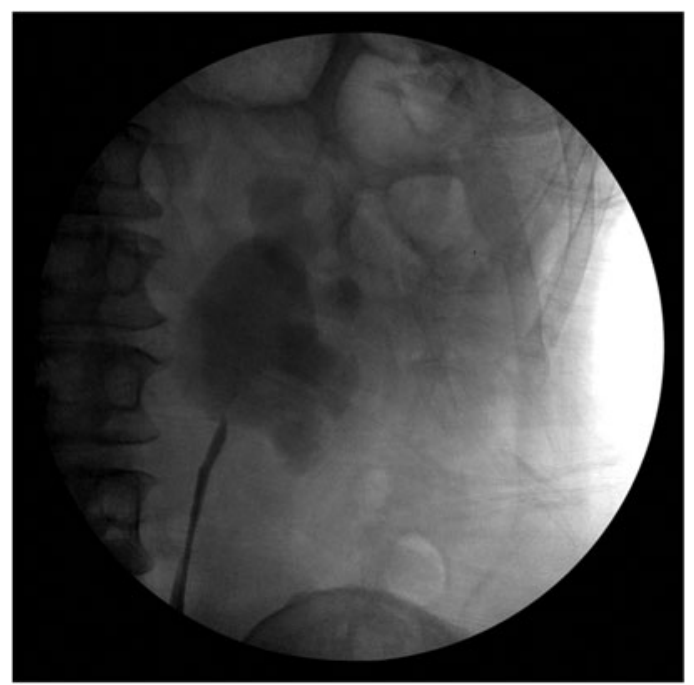

FIG. 1. Retrograde urogram shows a short-segment UPJO. UPJO, ureteropelvic junction obstruction.

(LP), ${ }^{9}$ the success rate in the endopyelotomy group dropped from $63 \%$ at 3 years to $41 \%$ at 10 years. However, the combined pyeloplasty group had lower-than-usual success rates, with $85 \%$ at 3 years to $75 \%$ at 10 years. There is overall consensus now that antegrade endopyelotomy continues to

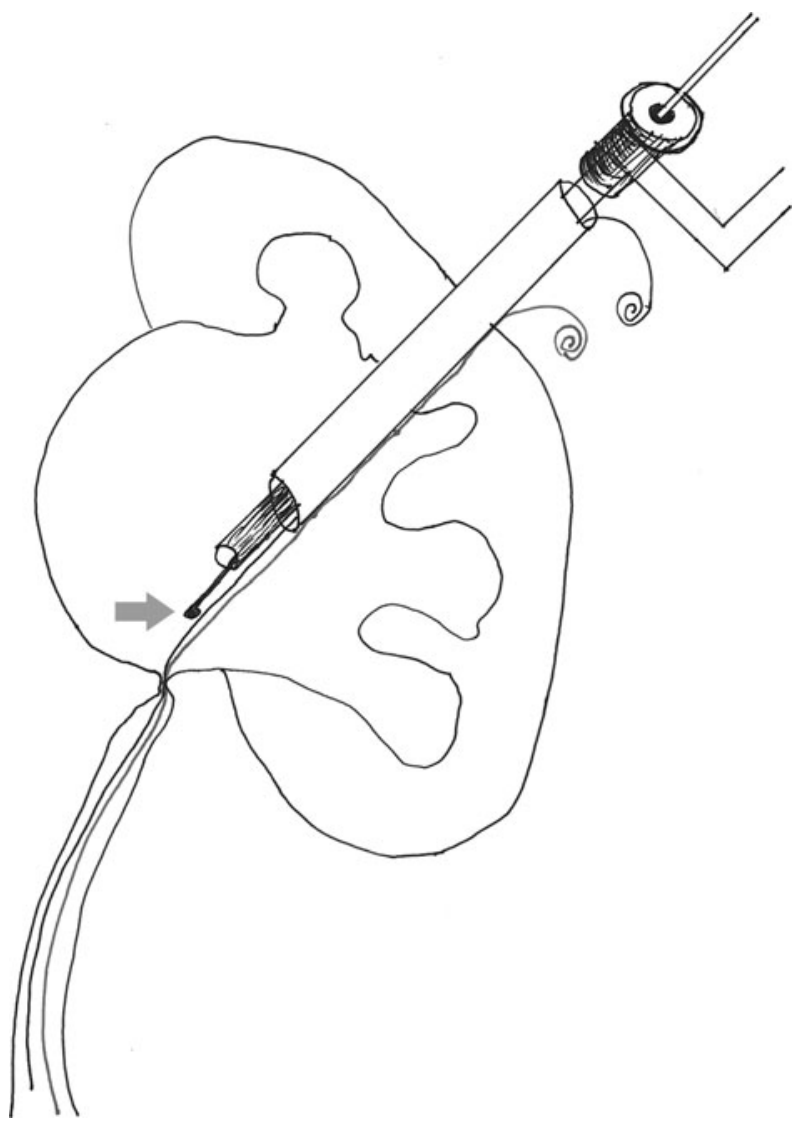

FIG. 2. Percutaneous antegrade access to the UPJ. Arrow depicts the cold knife that is used to incise the UPJO laterally. UPJ, ureteropelvic junction. be a viable option for short-length strictures, but success rate drops over time, as reported in those patients with long-term follow-up. ${ }^{9}$

Complications of antegrade endopyelotomy are similar to PCNL. In a summary of 27 published antegrade endopyelotomy series, ${ }^{8}$ the mean complication rate was $13.3 \%$ (range $0 \%-18 \%$ ), transfusion rate was $4.2 \%$ (range $0 \%-23 \%$ ), and success rate was $81 \%$ (range $61 \%-100 \%$ ). In a literature review of endopyelotomy complications, nephrostomy tract bleeding, arteriovenous fistula, pseudoaneurysms, ureteral necrosis, pneumothorax, lost guidewire, ureteral stent migration, urosepsis, and urinoma have all been reported. ${ }^{10}$

\section{Summary}

Percutaneous antegrade endopyelotomy is recommended in short-segment UPJO in adequately-functioning kidneys without a patulous renal pelvis. Careful follow-up to monitor success is recommended.

\section{Retrograde Endopyelotomy}

Retrograde endopyelotomy became feasible with advances in ureteroscopy, and with the experience and principles learned from the antegrade technique. The first published description of this technique was with Inglis and Tolley in 1986. ${ }^{11}$ At our institution in 1996, Thomas and colleagues presented their initial case series of 49 patients. ${ }^{12}$ In this series, the endopyelotomy was performed with an insulated $11.5 \mathrm{~F}$ rigid ureteroresectoscope with an electrocautery cutting element, followed by balloon dilatation of the UPJ. These patients were prestented, and the success rate was more than $90 \%$ in patients with a follow-up time greater than 6 months. Two patients underwent subsequent elective nephrectomy for poorly-functioning kidneys that did not improve despite retrograde endopyelotomy and prolonged ureteral stent drainage.

With the miniaturization of ureteroscopes, our group has ever since adapted our approach with $7.5 \mathrm{~F}$ semi-rigid and flexible ureteroscopes ${ }^{13}$ by using the Holmium laser for incising the UPJO. This allows ureteroscopy without prestenting (Fig. 3A, B). Balloon dilatation is recommended to ensure that all of the fibrous bands are incised. In the updated cohort of 133 patients who underwent retrograde endopyelotomy at our center between 1989 and 2001, recurrent obstruction requiring secondary treatment was noted in 32 (24\%) patients, especially in those with long-term follow-up data. Risk factors

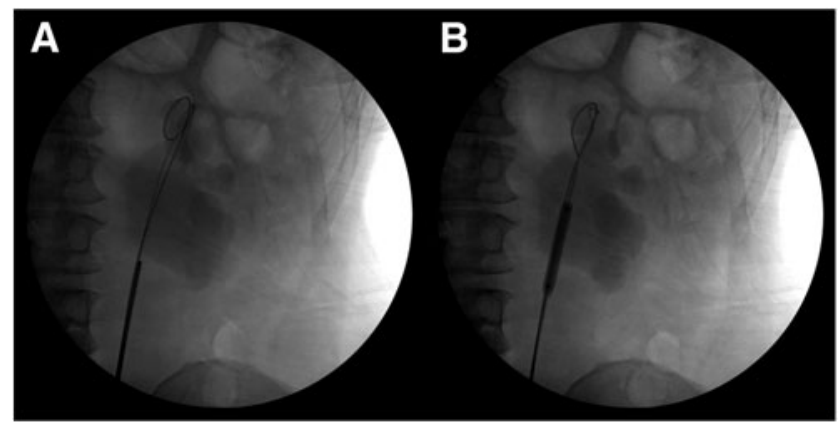

FIG. 3. (A) Rigid ureteroscopy and Holmium laser endopyelotomy. (B) Balloon dilatation of incised UPJO. 
associated with treatment failures included a split renal function of less than $20 \%$ in the ipsilateral kidney and a very dilated, patulous renal pelvis that did not improve postoperatively. Even with the use of the larger insulated ureteroresectoscope during the earlier part of the series, none of the patients developed ureteral strictures at long-term follow-up.

Because of the learning curve associated with ureteroscopy, other groups developed retrograde endopyelotomy with the use of a cutting electrode on a balloon (Acucise; Applied Medical, Rancho Santa Margarita, CA). With this technique, localization of the electrode depends on the quality of the fluoroscopic imaging during incision of the UPJ. In an early review of Acucise, success rates for this procedure in seven case series have been reported to range from $66 \%$ to $84 \% .{ }^{14}$ As the cutting was not done under direct vision, the major concern with this technique was the possibility of hemorrhage when incising the UPJO that is associated with aberrant crossing vessels. Later studies reported much lower success rates. ${ }^{15}$ The rates were as low as $25 \%$ in the cases when there was no contrast extravasation noted during the endopyelotomy to suggest whether a full-thickness incision was successfully performed to peri-ureteral fat. In such cases, Nakada and colleagues recommended conversion to flexible ureteroscopy with possible retrograde ureteroscopic endopyelotomy. ${ }^{14}$

Complications of ureteroscopic endopyelotomy, though similar to those encountered with ureteroscopy for urolithiasis, in general, have a less severe profile than antegrade endopyelotomy. In a retrospective comparison between ureteroscopic Holmium laser endopyelotomy and Acucise endopyelotomy, Ponsky and Streem reported their overall complication rates of both modalities as $10.1 \%$ and $25.7 \%$, respectively. ${ }^{16}$ In the ureteroscopy group, one patient required operative reintervention with temporary replacement of a ureteral stent for pain after initial stent removal. In the Acucise group, four patients required operative interventions: two with temporary replacement of ureteral stents, and two with selective arterial embolization of the lower pole vessel because of hemorrhage necessitating blood transfusions. Although the success rates were comparable $(74.3 \%$ and $77.8 \%$ for ureteroscopic and Acucise approaches, respectively), the authors preferred ureteroscopic endopyelotomy because of the hemorrhagic complications encountered with Acucise endopyelotomy. In comparison to other techniques for management of UPJO, Acucise endopyelotomy is currently very infrequently used.

\section{Summary}

Ureteroscopic endopyelotomy is recommended for shortsegment UPJO and is a direct extension of ureteroscopy skills. Today, this technique is recommended as a first-line intervention for recurrence of UPJO after definitive procedures such as open surgical, laparoscopic, or robotic pyeloplasty (RP).

\section{Percutaneous Endopyeloplasty}

This technique is an extension of antegrade endopyelotomy, with addition of endoscopic suturing of the UPJ in the Heineke-Mikulicz fashion. In the initial series from Cleveland Clinic in 2002, nine patients underwent this procedure, all with relief of symptoms and improved radiographic drainage in an early follow-up period of 4 months. ${ }^{17}$ An interval update with 15 patients showed $100 \%$ success with a follow-up period of 11.6 months. ${ }^{18}$ In an updated series of 55 patients, ${ }^{19}$ success was $90 \%$. Although a more recent publication from a different group ${ }^{20}$ described the technique with the use of a laparoscopic needle holder through the nephroscope, there have been no publications to date on the long-term outcomes of patients who have undergone this procedure. This technique has attained limited traction because of its complexity in suturing and the availability of less cumbersome techniques.

\section{Summary}

This technique, although initially promising, has not been used widely for UPJO management.

\section{Laparoscopic Pyeloplasty}

With the development of laparoscopic techniques in urology in the early 1990s, LP was added to the armamentarium of procedures offered by urologists for management of UPJO. In adult patients between 1999 and 2002, Klingler and colleagues retrospectively reported a comparison between 40 transperitoneal LPs and 15 retroperitoneal OP procedures. ${ }^{21}$ Differences between LP and OP included mean length of hospital stay (5.9 days vs 13.4 days) and mean length of skin incision $(4.1 \mathrm{~cm}$ vs $23.1 \mathrm{~cm})$. Invariably, hospital stays are known to be dependent on regional policies and expectations of hospitalization. Success rates for the dismembered LPs and OPs were comparable at $96 \%$ and $93.3 \%$, respectively. The mean follow-up time was 23.4 months for laparoscopy and 21.9 for open pyeloplasty. A significant decrease in postoperative pain and mean tramadol requirement was seen for LPs (190 mg vs $370 \mathrm{mg}$ for OPs). Although there were no intraoperative complications, postoperatively $17.5 \%$ of the LP patients experienced complications compared with $40 \%$ in the open group. Postoperative complications included anastomotic strictures, re-operation, deep venous thrombosis, clot retention, urinoma, recurrent urinary tract infection (UTI), and abdominal wall herniae.

Bansal and colleagues reported on a prospective, randomized, single-center trial comparing 34 OPs and 28 transperitoneal LPs in adults between 2004 and 2007 with mean followup of 33.5 and 34.5 months, respectively. ${ }^{22}$ The mean operative times were 122 and 244 minutes for OP and LP, respectively. The authors noted improvement in operative time with increased laparoscopic experience, resulting in a mean operative time of 202 in the last five LP cases. Less duration and use of analgesia were required in the LP group. The mean hospital stay was shorter by a mean of $\sim 5$ days in favor of the LP group.

In a retrospective study from Zhang and colleagues, 56 retroperitoneal LPs were compared with 40 retroperitoneal OPs in adults. ${ }^{23}$ Significant differences between LP and OP were noted for operative time (80 minutes $v s 120$ minutes), estimated blood loss $(10 \mathrm{~mL}$ vs $150 \mathrm{~mL})$, return of bowel function (1 days $v s 2$ days), diclofenac analgesia requirement (75 mg vs $150 \mathrm{mg})$, incision length $(3.5 \mathrm{~cm}$ vs $21 \mathrm{~cm})$, and length of hospital stay ( 7 days $v s 9$ days). No intraoperative complications occurred in either group. There was no significant difference in success rate between the LP and OP groups (98.2\% vs $97.5 \%$ ). The mean follow-up was 30.2 and 23.4 months for LP and OP, respectively. This further 
illustrates the significant decrease in morbidity of minimallyinvasive procedures such as LP over OP.

Complications of LP include those often seen in other types of laparoscopic urologic procedures. Intraoperative complications $(2 \%-2.3 \%)$ include ligation of the lower pole artery, loss of a needle, hypercapnea, cutting the ureteral stent, colonic injury, and port site bleeding. ${ }^{24}$ The conversions from LP to open pyeloplasty were reported to be due to inability to access the UPJ or to complete the UPJ anastomosis. Postoperative complications $(12.9 \%-15.8 \%)$ included urine leak, hematoma formation, colonic lesion, and stone formation. Recurrent UPJO requiring secondary procedures ranged from $3.5 \%$ to $4.8 \%$. These complications have been largely attributed to the learning curve associated with LP and the challenges with mastering suturing proficiency.

\section{Summary}

LP is a viable option for using minimally-invasive techniques for treating UPJO, with excellent success rates. However, LP has been limited to major academic centers, to those facile with laparoscopic suturing techniques, and where there is no access to the surgical robot. The challenges encountered with mastering pure laparoscopic suturing techniques have essentially been solved with the introduction of robotic technology.

\section{Robotic Pyeloplasty}

With the introduction of robotic surgery utilizing a computer interface in 2002, procedures such as RP requiring intracorporeal suturing gained widespread acceptance because of the articulation and stabilization provided by the robotic instrumentation that mimicked hand movements. RP offers the advantages of laparoscopy in terms of morbidity and, in addition, offers a quicker learning curve with the potential of improved results due to enhanced three-dimensional visualization. ${ }^{25}$ In regions with access to robotic technology, there has been a rapid acceptance and use of RP. ${ }^{26}$

RP essentially utilizes the same principles and techniques and has had results that are almost identical to those reported in older OP series. Most RP series include the AndersonHynes dismembered technique (Fig. 4) with excision of the pathologic UPJ segment, removal of any concomitant stones,

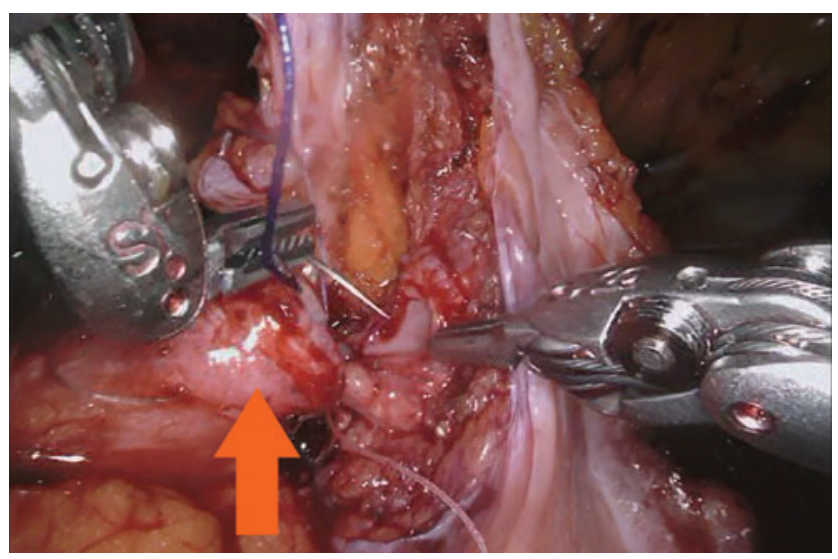

FIG. 4. Robotic pyeloplasty. Needle about to be placed into the renal pelvis. Arrow points to the ureter. and transposition of the collecting system in relationship to the crossing vessels, if present and if needed. ${ }^{27}$

Schwentner and colleagues performed 92 transperitoneal RPs with a mean follow-up of 39.1 months and with a $96.7 \%$ success rate. ${ }^{28}$ The mean operative time, including robotic setup, was 108 minutes. The mean hospital stay was 4.57 days. Failures were attributed to hemorrhage into the collecting system and extravasation early after surgery. No late complications were noted. Three patients required additional procedures. The success was defined by imaging with intravenous urography and MAG-3 nuclear medicine renal functional study and resolution of pain. The success rate, similar to OP, was attributed to the quality of the ureteropelvic anastomosis allowed by the robot's functionality.

A retrospective study by Mufarrij and colleagues included 104 patients who were treated with dismembered RP from three university medical centers. ${ }^{29}$ These include 117 primary and 23 secondary repairs. Thirteen patients underwent simultaneous stone extraction, and five patients had solitary kidneys. The mean operative time was 217 minutes, estimated blood loss was $59.4 \mathrm{~mL}$, mean length of stay was 2.1 days, and mean follow-up was 29 months. Success was noted in $95.7 \%$ patients, and it was defined as radiographic resolution of obstruction on first postoperative diuretic renal scan or excretory urogram. Complication rates, $7.1 \%$ major and $2.9 \%$ minor, were reported. The 10 major complications included stent migration, gluteal compartment syndrome, obstruction from a UPJ blood clot, worsening hydronephrosis, and pyelonephritis requiring stent exchange on postoperative day 5 . There were four minor complications, including a febrile UTI, minor splenic laceration, and two urine leaks. All patients who experienced a complication recovered after appropriate minimally-invasive intervention with ultimate demonstration of successful RP.

Introduction of RP in the adult population has led to its percolation to the pediatric patient group, as pediatric urologists are increasingly implementing robotic techniques into their practice. ${ }^{30}$ Moreover, with experience and pursuing published techniques, urologists can manage patients with concomitant kidney stones and those who have failed prior attempts at repairing their UPJO. ${ }^{27}$

\section{Summary}

RP has supplanted other menu options for management of UPJO, when robotic equipment is available. Success rates are similar to those reported with OP. RP mimics OP without the morbidity.

\section{Conclusions}

Over the past three decades, management of UPJO has gone through an evolutionary process. Introduction of newer technologies, techniques, and lessons learned from the past have shaped the course of UPJO management. RP comes the closest to duplicating open surgical techniques, safe tissue handling, and outcomes. Clearly, the techniques that have been accepted and utilized are those that are easy to duplicate, and outcomes that match those reported with OP, which is still considered a gold standard for comparison.

\section{Author Disclosure Statement}

No competing financial interests exist. 


\section{References}

1. Wickham JE, Kellet MJ. Percutaneous pyelolysis. Eur Urol 1983;9:122-124.

2. Ramsay JW, Miller RA, Kellett MJ, et al. Percutaneous pyelolysis: Indications, complications and results. Br J Urol 1984;56:586-588.

3. Badlani G, Eshghi M, Smith AD. Percutaneous surgery for ureteropelvic junction obstruction (endopyelotomy): Technique and early results. J Urol 1986;135:26-28.

4. Sampaio FJ, Favorito LA. Ureteropelvic junction stenosis: Vascular anatomical background for endopyelotomy. J Urol 1993;150:1787-1791.

5. Bernardo NO, Smith AD. Percutaneous endopyelotomy. Urology 2000;56:322-327.

6. Savage SJ, Streem SB. Simplified approach to percutaneous endopyelotomy. Urology 2000;56:848-850.

7. Gerber GS, Lyon ES. Endopyelotomy: Patient selection, results, and complications. Urology 1994; 43:2-10.

8. Gupta M, Tuncay OL, Smith AD. Open surgical exploration after failed endopyelotomy: A 12-year perspective. J Urol 1997;157:1613-1618.

9. Dimarco DS, Gettman MT, McGee SM, et al. Long-term success of antegrade endopyelotomy compared with pyeloplasty at a single institution. J Endourol 2006;20:707712.

10. Bellman GC. Complications of endopyelotomy. J Endourol 1996; 10:177-181.

11. Inglis JA, Tolley DA. Ureteroscopic pyelolysis for pelviureteric junction obstruction. Br J Urol 1986;58:250 252.

12. Thomas R, Monga M, Klein EW. Ureteroscopic retrograde endopyelotomy for management of ureteropelvic junction obstruction. J Endourol 1996;10:141-145.

13. Mendez-Torres FR, Urena R, Thomas R. Retrograde ureteroscopic endopyelotomy. Urol Clin North Am 2004;31: 99-106.

14. Nakada SY, Pearle MS, Clayman RV. Acucise endopyelotomy: Evolution of a less-invasive technology. J Endourol 1996;10:133-139.

15. Biyani CS, Minhas S, el Cast J, et al. The role of Acucise endopyelotomy in the treatment of ureteropelvic junction obstruction. Eur Urol 2002;41:305-310.

16. Ponsky LE, Streem SB. Retrograde endopyelotomy: A comparative study of hot-wire balloon and ureteroscopic laser. J Endourol 2006;20:823-826.

17. Gill IS, Desai MM, Kaouk JH, et al. Percutaneous endopyeloplasty: Description of new technique. J Urol 2002; 168:2097-2102.

18. Desai MM, Desai MR, Gill IS. Endopyeloplasty versus endopyelotomy versus laparoscopic pyeloplasty for primary ureteropelvic junction obstruction. Urology 2004;64: $16-21$.

19. Stein RJ, Gill IS, Desai MM. Comparison of surgical approaches to ureteropelvic junction obstruction: Endopyeloplasty versus endopyelotomy versus laparoscopic pyeloplasty. Curr Urol Rep 2007;8:140-149.
20. Lezrek M, Bazine K, Moufid K, et al. A more "conventional" way to perform percutaneous endopyeloplasty: A feasibility study. Urology 2012;79:227-230.

21. Klingler HC, Remzi M, Janetschek G, et al. Comparison of open versus laparoscopic pyeloplasty techniques in treatment of uretero-pelvic junction obstruction. Eur Urol 2003;44:340-345.

22. Bansal P, Gupta A, Mongha R, et al. Laparoscopic versus open pyeloplasty: Comparison of two surgical approachesA single centre experience of three years. Indian J Surg 2011;73:264-267.

23. Zhang X, Li HZ, Ma X, et al. Retrospective comparison of retroperitoneal laparoscopic versus open dismembered pyeloplasty for ureteropelvic junction obstruction. J Urol 2006;176:1077-1080.

24. Rassweiler JJ, Teber D, Frede T. Complications of laparoscopic pyeloplasty. World J Urol 2008;26:539-547.

25. Autorino R, Eden C, El-Ghoneimi A, et al. Robot-assisted and laparoscopic repair of ureteropelvic junction obstruction: A systematic review and meta-analysis. Eur Urol 2014; 65:430-452.

26. Jacobs BL, Kaufman SR, Morgenstern H, et al. Trends in the treatment of adults with ureteropelvic junction obstruction. J Endourol 2013;27:355-360.

27. Atug F, Castle EP, Burgess SV, et al. Concomitant management of renal calculi and pelvi-ureteric junction obstruction with robotic laparoscopic surgery. BJU Int 2005;96: 1365-1368.

28. Schwentner C, Pelzer A, Neururer R, et al. Robotic AndersonHynes pyeloplasty: 5-year experience of one centre. BJU Int 2007;100:880-885.

29. Mufarrij PW, Woods M, Shah OD, et al. Robotic dismembered pyeloplasty: A 6-year, multi-institutional experience. J Urol 2008;180:1391-1396.

30. Atug F, Woods M, Burgess SV, et al. Robotic assisted laparoscopic pyeloplasty in children. J Urol 2005;174:14401442.

\section{Address correspondence to: Raju Thomas, MD, FACS, MHA Department of Urology \\ Tulane University School of Medicine 1430 Tulane Avenue, SL-42 New Orleans, LA 70112 \\ E-mail:rthomas@tulane.edu}

$$
\begin{aligned}
& \text { Abbreviations Used } \\
& \text { LP }=\text { laparoscopic pyeloplasty } \\
& \text { OP }=\text { open pyeloplasty } \\
& \text { PCNL }=\text { percutaneous nephrostolithotripsy } \\
& \text { RP }=\text { robotic pyeloplasty } \\
& \text { UPJ }=\text { ureteropelvic junction } \\
& \text { UPJO }=\text { ureteropelvic junction obstruction } \\
& \text { UTI }=\text { urinary tract infection }
\end{aligned}
$$

